Microwave beamforming for non-invasive patient-specific hyperthermia treatment of pediatric brain cancer

This article has been downloaded from IOPscience. Please scroll down to see the full text article.

2011 Phys. Med. Biol. 562743

(http://iopscience.iop.org/0031-9155/56/9/007)

View the table of contents for this issue, or go to the journal homepage for more

Download details:

IP Address: 128.104.181.78

The article was downloaded on 25/04/2011 at 20:58

Please note that terms and conditions apply. 


\title{
Microwave beamforming for non-invasive patient-specific hyperthermia treatment of pediatric brain cancer
}

\author{
Matthew J Burfeindt ${ }^{1}$, Earl Zastrow ${ }^{1}$, Susan C Hagness ${ }^{1}$, \\ Barry D Van Veen ${ }^{1}$ and Joshua $\mathbf{E}$ Medow $^{2,3}$ \\ ${ }^{1}$ Department of Electrical and Computer Engineering, University of Wisconsin-Madison, WI, \\ USA \\ ${ }^{2}$ Department of Neurological Surgery, University of Wisconsin-Madison, WI, USA \\ ${ }^{3}$ Department of Biomedical Engineering, University of Wisconsin-Madison, WI, USA \\ E-mail: bmatthew@wisc.edu, earl.zastrow@ieee.org, hagness@engr.wisc.edu, \\ vanveen@engr.wisc.edu and medow@neurosurg.wisc.edu
}

Received 8 January 2011, in final form 6 March 2011

Published 5 April 2011

Online at stacks.iop.org/PMB/56/2743

\begin{abstract}
We present a numerical study of an array-based microwave beamforming approach for non-invasive hyperthermia treatment of pediatric brain tumors. The transmit beamformer is designed to achieve localized heating - that is, to achieve constructive interference and selective absorption of the transmitted electromagnetic waves at the desired focus location in the brain while achieving destructive interference elsewhere. The design process takes into account patient-specific and target-specific propagation characteristics at $1 \mathrm{GHz}$. We evaluate the effectiveness of the beamforming approach using finite-difference time-domain simulations of two MRI-derived child head models from the Virtual Family (IT'IS Foundation). Microwave power deposition and the resulting steady-state thermal distribution are calculated for each of several randomly chosen focus locations. We also explore the robustness of the design to mismatch between the assumed and actual dielectric properties of the patient. Lastly, we demonstrate the ability of the beamformer to suppress hot spots caused by pockets of cerebrospinal fluid (CSF) in the brain. Our results show that microwave beamforming has the potential to create localized heating zones in the head models for focus locations that are not surrounded by large amounts of CSF. These promising results suggest that the technique warrants further investigation and development.
\end{abstract}

(Some figures in this article are in colour only in the electronic version) 


\section{Introduction}

The goal of hyperthermia treatment is to elevate the temperature of a tumor above a certain threshold (commonly $42^{\circ} \mathrm{C}$ ) for an extended period of time, thereby weakening or destroying the cancer cells, while maintaining safe temperatures in healthy tissues. Hyperthermia treatment has been shown to be an effective adjuvant to other treatment modalities for a variety of cancers (e.g., Overgaard et al 1995, Kapp 1996, Van der Zee et al 2000, Harima et al 2001, Sneed et al 2004). For brain tumors, favorable outcomes have been achieved after interstitial hyperthermia treatment (e.g., Winter et al 1985, Tanaka et al 1987, Sneed et al 1998).

Inducing localized hyperthermia in the pediatric brain via a microwave transmit beamforming array has potential advantages relative to current treatment methods. Microwave beamforming is a non-invasive process and thus may allow the treatment of brain tumors that cannot be treated safely by surgical excision. Microwave radiation is also non-ionizing, unlike the gamma radiation often employed in radiation therapy. Using ionizing radiation to treat brain tumors in children carries risk, as it is linked with developmental disorders (Pollack 1999, Duffner 2004). In a study by Packer et al (1989), children who received cranial radiation treatment and were 7 years of age or younger at diagnosis experienced a mean decline of 25 points in full-scale IQ 2 years after treatment. Duffner et al (1985) found that 86\% of children who received cranial radiation treatment experienced growth hormone deficiencies in the year following treatment.

Non-invasive focused microwave systems for the brain have previously been studied using numerical simulations. Dunn et al (1996) investigated the use of a spherical array of sources to focus electromagnetic energy in a numerical human head phantom. Gouzouasis et al (2007) conducted simulations of an ellipsoidal reflecting cavity for focusing energy into a numerical human head phantom. The latter group also developed a prototype of this cavity and has tested it on a physical phantom (Karanasiou et al 2008). To the best of our knowledge, neither of these techniques incorporates patient-specific propagation data into their design.

The widespread availability of MRI-derived, anatomically realistic human phantoms and the relative ease with which they are constructed and used in full-wave electromagnetic simulations make it possible to design microwave beamformers based on patient-specific and focus-location-specific propagation data. Numerical studies of beamforming for focusing in the breast have recently been conducted (Zastrow et al 2010). Using such an approach for the brain presents different challenges, as most matter in the brain is lossy high-watercontent tissue. In contrast, one of the major constituents of the breast is lower-loss adipose tissue. The existence of cerebrospinal fluid (CSF) in the brain further complicates selective microwave heating. CSF is highly conductive in comparison to other brain tissue and is also not cooled by the blood supply. Therefore, it is prone to heat accumulation under microwave illumination, resulting in unsafe temperatures. Because of the challenges imposed by highwater-content tissue and CSF, the demonstrated feasibility of microwave beamforming for breast hyperthermia does not guarantee feasibility for brain hyperthermia.

In this paper, we present a numerical study of a microwave beamforming approach for noninvasively inducing localized hyperthermia in the pediatric brain. Adult brains tend to have a higher volume of CSF than pediatric brains and thus represent a more challenging treatment scenario. Given this challenge and the risks of radiation therapy in children, this feasibility study is directed to treatment of pediatric brain cancer. The beamformer is designed to focus microwave energy at a specific location within the brains of two numerical MRI-derived child head phantoms. We evaluate the beamformer's performance using electromagnetic and thermal simulations of the phantoms. Selective heating performance at $1 \mathrm{GHz}$ is evaluated for 
a set of randomly chosen focus locations in the brain. In addition, we explore the robustness of beamforming to modest mismatch between the actual and assumed dielectric properties of the patient. The ability of the beamforming approach to suppress unwanted hot spots due to CSF in the brain is also demonstrated.

The remainder of the paper is organized as follows. Section 2 describes the transmit beamforming strategy as well as the numerical models we use in the study. The results of the study are presented and discussed in section 3. Concluding statements are made in section 4.

\section{Models and methods}

\subsection{Transmit beamforming}

Our transmit beamforming goal is to achieve selective absorption of microwave energy at a desired location in the brain. The beamformer is designed so that narrowband signals transmitted from the antennas in the array achieve constructive interference and increased energy deposition at the desired focus location. We may also suppress energy deposition at other locations in the brain by designing the beamformer to achieve destructive interference at those locations.

The beamformer comprises one complex channel weight for each antenna. Each channel weight determines the magnitude and phase of the input signal to the corresponding antenna. The channel weight for the $i$ th channel is denoted by $w_{i}$. Define the vector $\mathbf{w}$ as

$$
\mathbf{w}=\left[w_{1} w_{2} \ldots w_{N}\right]^{\mathrm{H}},
$$

where the superscript $\mathrm{H}$ denotes conjugate transpose.

For a given location in the head $\mathbf{r}$, each antenna has a complex transfer function that relates the signal fed to the antenna to the electric field phasor seen at $\mathbf{r}$. Denote the channel transfer function for the $i$ th channel and location $\mathbf{r}$ by $h_{i}(\mathbf{r})$. Define the vector $\mathbf{h}(\mathbf{r})$ as

$$
\mathbf{h}(\mathbf{r})=\left[h_{1}(\mathbf{r}) h_{2}(\mathbf{r}) \ldots h_{N}(\mathbf{r})\right]^{\mathrm{T}} .
$$

For this study, we use the copolarized component of the electric field to determine $\mathbf{h}(\mathbf{r})$. In a clinical setting, $\mathbf{h}(\mathbf{r})$ is not known exactly for all $\mathbf{r}$ of interest, so a patient-specific approximation of $\mathbf{h}(\mathbf{r})$ is needed. This approximation may be obtained by acquiring an MRI of the patient's head, assigning dielectric properties to each tissue type in the MRI, and conducting electromagnetic simulations of the MRI-derived head model. The latter step in this clinical treatment planning protocol is identical to that used to obtain $\mathbf{h}(\mathbf{r})$ in this study involving virtual patients.

Suppose that the desired focus is located at $\mathbf{r}_{f}$. Also suppose that there are $M$ locations $\left(\mathbf{r}_{s 1}, \mathbf{r}_{s 2}, \ldots, \mathbf{r}_{s M}\right)$ where we wish to suppress energy deposition. We accomplish our beamformer design goal by choosing $\mathbf{w}$ to solve the following optimization problem:

$$
\mathbf{w}=\arg \max _{\mathbf{w}} \frac{\mathbf{w}^{\mathrm{H}} \mathbf{h}\left(\mathbf{r}_{f}\right) \mathbf{h}^{\mathrm{H}}\left(\mathbf{r}_{f}\right) \mathbf{w}}{\mathbf{w}^{\mathrm{H}}\left\{\mathbf{S}\left(\mathbf{r}_{f}\right)+\sum_{i=1}^{M} \alpha_{i} \mathbf{h}\left(\mathbf{r}_{s i}\right) \mathbf{h}^{\mathrm{H}}\left(\mathbf{r}_{s i}\right)\right\} \mathbf{w}} .
$$

The scalars $\left(\alpha_{1}, \alpha_{2}, \ldots, \alpha_{M}\right)$ determine the relative importance of suppressing energy deposition at locations $\left(\mathbf{r}_{s 1}, \mathbf{r}_{s 2}, \ldots, \mathbf{r}_{s M}\right)$. We define $\mathbf{S}\left(\mathbf{r}_{f}\right)$ to be an $N \times N$ diagonal matrix, where $S_{i i}\left(\mathbf{r}_{f}\right)=\left|h_{i}\left(\mathbf{r}_{f}\right)\right|$. Defining $\mathbf{S}\left(\mathbf{r}_{f}\right)$ in this way penalizes solutions where the magnitudes of the $w_{i}$ differ much from each other. For example, it is straightforward to show that when $\alpha_{i}=0, i=1,2, \ldots, M$, this choice results in $\left|w_{i}\right|=1$. Keeping the output power across the array relatively constant prevents a concentration of heating at any point on the head surface. The solution to (1) is $\mathbf{w}=\left\{\mathbf{S}\left(\mathbf{r}_{f}\right)+\sum_{i=1}^{M} \alpha_{i} \mathbf{h}\left(\mathbf{r}_{s i}\right) \mathbf{h}^{\mathrm{H}}\left(\mathbf{r}_{s i}\right)\right\}^{-1} \mathbf{h}\left(\mathbf{r}_{f}\right)$. 


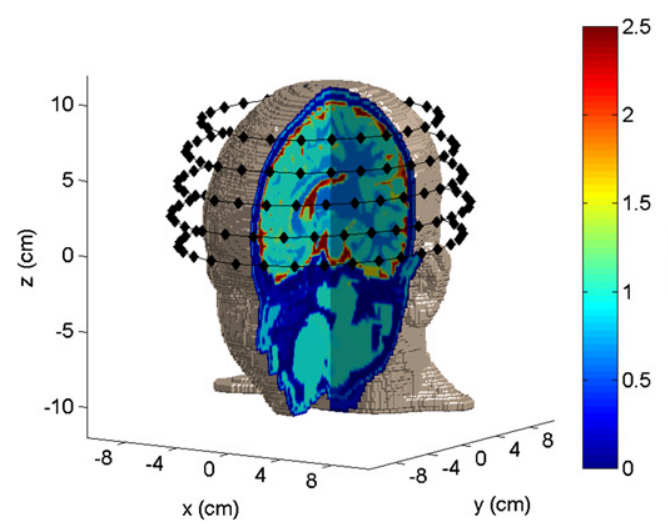

(a)

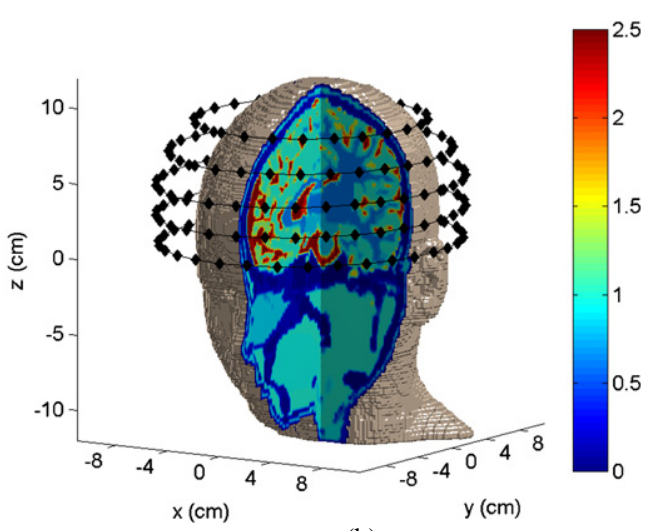

(b)

Figure 1. Numerical models of (a) the 6 year old patient and (b) the 11 year old patient (Virtual Family, IT'IS Foundation). The cross-sections show the interior effective conductivity in $\mathrm{S} \mathrm{m}^{-1}$. The locations of the sources are signified by the black dots on the rings encircling the phantoms.

\subsection{Numerical models for beamformer design and performance evaluation}

Numerical phantoms of a 6 year old male and an 11 year old female are used as virtual patients in this study. The phantoms are from the Virtual Family available through the IT'IS Foundation (Zurich, Switzerland) ${ }^{4}$. The anatomically realistic 3D phantoms are derived from MRI scans of human subjects. The phantoms are segmented into a number of tissue types (up to 84 in the whole body models). Each tissue type is assigned appropriate dielectric and thermal properties. The phantoms used here have a $1 \mathrm{~mm}$ resolution. Treatment planning for the virtual patients is performed by determining $\mathbf{h}(\mathbf{r})$ using finite-difference time-domain (FDTD) simulations (see Taflove and Hagness 2005) of the human head models and designing the beamformer weights according to (1). Note that this approach is an ideal approximation to the clinical scenario because the model used to derive the propagation function $\mathbf{h}(\mathbf{r})$ is identical to the model used to evaluate the beamformer's performance. In a clinical setting, there will be differences between the MRI-derived propagation model and the actual propagation environment of the patient's head. These differences arise from mismatch between the assumed and actual dielectric properties of the tissues in the patient's head.

2.2.1. Electromagnetic model for beamformer design. Electromagnetic (EM) FDTD simulations in this study are conducted using SEMCAD X version 14 from Schmid and Partner Engineering AG (SPEAG). The head phantoms and the surrounding antenna array are illustrated in figure 1. The virtual patients are oriented upright in the positive $z$-direction. The array elements in the simulations are $1 \mathrm{~mm}$ long, $z$-directed voltage sources with internal resistance $50 \Omega$. These sources are distributed on elliptical rings encircling the phantoms. The plane of each ring is parallel to the $x y$-plane. Five rings of antennas are separated by a vertical spacing of $2 \mathrm{~cm}$, with the bottom ring $2 \mathrm{~cm}$ above eye level. The in-plane spacing between sources on each ring is $2 \mathrm{~cm}$. The major and minor axes of each ring are chosen so that the minimum in-plane distance from any source to the skin surface is $2 \mathrm{~cm}$. A total of 147 sources are used for the 6 year old patient, while 152 sources are used for the 11 year old patient. The slight increase in the number of sources between phantoms is due to the slightly larger extent of the 11 year old patient's head in some planes. A de-ionized (DI) water half-space extends

4 See http://www.itis.ethz.ch/services/human-and-animal-models/human-models/ 
upward from a plane located $7 \mathrm{~mm}$ below the bottom ring of sources. The DI water provides effective coupling of microwave power into the head interior. Below the DI water half-space, the surrounding medium is air.

The frequency of operation for the EM simulations is $1 \mathrm{GHz}$. This frequency is chosen to balance the need for high spatial resolution with the need for an adequate penetration depth. The relative permittivity, $\epsilon_{r}$, and effective conductivity, $\sigma_{\text {eff }}$, at $1 \mathrm{GHz}$ were taken from the report by Gabriel (1996) $)^{5}$. Brain structures for which dielectric data were not reported by Gabriel (1996), such as the medulla oblongata, pons, and midbrain, were assumed to have dielectric properties averaged between gray matter and white matter.

The propagation vector $\mathbf{h}(\mathbf{r})$ for any $\mathbf{r}$ of interest is found via FDTD simulation using reciprocity. A source is placed at $\mathbf{r}$ and the simulation is run until the sinusoidal steady state is reached. The resulting phasors recorded across the beamforming array are then used as an approximation to $\mathbf{h}(\mathbf{r})$.

2.2.2. Electromagnetic and thermal model for performance evaluation. The electromagnetic performance evaluation simulation is conducted with $\mathbf{w}$ feeding the array. The resulting steadystate heating potential, $Q(\mathbf{r})$, inside the head is computed and saved for use in the thermal FDTD simulation. The thermal simulation is also conducted using SEMCAD X. The thermal model solves the well-known Pennes bioheat equation, given by

$C_{p}(\mathbf{r}) \rho(\mathbf{r}) \frac{\partial T(\mathbf{r})}{\partial t}=\nabla \cdot(K(\mathbf{r}) \nabla T(\mathbf{r}))+A_{0}(\mathbf{r})+Q(\mathbf{r})-B(\mathbf{r})\left(T(\mathbf{r})-T_{B}\right)$,

where $C_{p}$ is the specific heat, $\rho$ is the mass density, $K$ is the thermal conductivity, $A_{0}$ is the metabolic heat production, $Q$ is the electromagnetic heating potential calculated from the EM FDTD simulation, $B$ is a constant representing heat exchange due to capillary blood perfusion, and $T_{B}$ is the blood temperature (set to $37^{\circ} \mathrm{C}$ ). Thermal properties for each tissue type were taken from Duck (1990) and Bernardi et al (2003). Due to the dearth of data regarding metabolic heat generation in the literature, $A_{0}$ is assumed to be proportional to the blood perfusion. This follows the convention used by Gordon et al (1976) and Bernardi et al (2003).

The air and DI water surrounding the head are assumed to be at $15{ }^{\circ} \mathrm{C}$. The DI water therefore acts as a superficial cooling medium. A convective boundary condition is applied at the skin-water interface with a convective coefficient of $200 \mathrm{~W}\left(\mathrm{~m}^{2} \mathrm{~K}\right)^{-1}$. This value is more conservative than the value of $300 \mathrm{~W}\left(\mathrm{~m}^{2} \mathrm{~K}\right)^{-1}$ used by Converse et al (2006), which was found by extrapolating the experimental results for a cooling system used in ultrasound vasectomy. The convective coefficient for the air-skin interface is set to $10 \mathrm{~W}\left(\mathrm{~m}^{2} \mathrm{~K}\right)^{-1}$.

All body tissue temperatures are initialized to $37{ }^{\circ} \mathrm{C}$. A preliminary cooling period of $100 \mathrm{~s}$ is simulated. The heating potential $Q(\mathbf{r})$ is then applied until steady state is reached.

\section{Simulation results and discussion}

\subsection{6 year old patient, ideal beamformer with no suppression points}

First we consider beamforming performance for eight randomly chosen focus locations in the numerical models of the 6 year old patient. The focus locations were constrained to be separated by at least $2 \mathrm{~cm}$ and to be at least $1 \mathrm{~cm}$ deep in the brain. The focus locations were also constrained to have at most $0.1 \mathrm{~mL}$ of CSF within a $1.5 \mathrm{~cm}$ radius. The CSF volume constraint was included to avoid focusing directly onto a large CSF pocket. Focusing near a large CSF pocket causes the heating zone to move from the desired focus location to the

5 A compilation can be found at http://www.fcc.gov/oet/rfsafety/dielectric.html. 
Table 1. Coordinates of the focus locations in the model of the 6 year old patient.

\begin{tabular}{lrrl}
\hline Point & $x(\mathrm{~cm})$ & $y(\mathrm{~cm})$ & $z(\mathrm{~cm})$ \\
\hline $\mathbf{r}_{f 1}$ & -2.2 & -4.0 & 5.5 \\
$\mathbf{r}_{f 2}$ & -2.2 & 1.8 & 7.9 \\
$\mathbf{r}_{f 3}$ & -2.2 & -3.4 & 2.9 \\
$\mathbf{r}_{f 4}$ & 3.2 & 3.1 & 6.3 \\
$\mathbf{r}_{f 5}$ & -2.2 & 3.8 & 6.7 \\
$\mathbf{r}_{f 6}$ & 2.7 & -1.8 & 5.8 \\
$\mathbf{r}_{f 7}$ & 1.5 & -4.4 & 2.7 \\
$\mathbf{r}_{f 8}$ & -2.1 & -0.5 & 6.2 \\
\hline
\end{tabular}

location of the pocket. No suppression points were used, resulting in an equal transmit power distribution across the array.

We define the following nomenclature to describe the simulation results.

$\mathbf{r}_{f i}$ : the $i$ th focus location,

$\mathbf{r}_{p i}$ : the location of peak temperature obtained when focusing at $\mathbf{r}_{f i}$,

$P$ : the total output power of the array,

$T(\mathbf{r})$ : the steady-state temperature distribution.

The coordinates for the focus locations are given in table 1. We refer to the tissue within the $42{ }^{\circ} \mathrm{C}$ contour surrounding $\mathbf{r}_{p i}$ as the heating zone. Any points exterior to the $41{ }^{\circ} \mathrm{C}$ contour surrounding $\mathbf{r}_{p i}$ where $T(\mathbf{r})>41{ }^{\circ} \mathrm{C}$ are termed hot spots. We scaled $P$ to maximize $T\left(\mathbf{r}_{f i}\right)$ such that the hottest point exterior to the $41{ }^{\circ} \mathrm{C}$ contour had a temperature $T(\mathbf{r})=40.95 \pm$ $0.15^{\circ} \mathrm{C}$. The extents of the heating zone in the $x$-, $y$-, and $z$-directions are referred to as $L_{x}$, $L_{y}$, and $L_{z}$, respectively.

Figure 2 shows three orthogonal cross-sectional views of the thermal distributions for two representative focus locations, $\mathbf{r}_{f 1}$ and $\mathbf{r}_{f 2}$. The focus location $\mathbf{r}_{f 1}$ is in the front left of the brain, while $\mathbf{r}_{f 2}$ is in the central left. The orthogonal cuts pass through $\mathbf{r}_{p 1}$ and $\mathbf{r}_{p 2}$, respectively. The focus locations are projected onto each cross-sectional plane and are marked with a white cross-hair. The heating zone is shown by the solid lines, while the dashed lines correspond to the $41{ }^{\circ} \mathrm{C}$ contour. For $\mathbf{r}_{f 1}, T\left(\mathbf{r}_{f 1}\right)=44.0{ }^{\circ} \mathrm{C}, L_{z}=28, L_{x}=15$, and $L_{y}=15$ $\mathrm{mm}$. For $\mathbf{r}_{f 2}, T\left(\mathbf{r}_{f 2}\right)=42.2^{\circ} \mathrm{C}, L_{z}=14, L_{x}=10$, and $L_{y}=9 \mathrm{~mm}$.

Figure 3 relates $T\left(\mathbf{r}_{f i}\right)$ and $T\left(\mathbf{r}_{p i}\right)$ to $L_{z}$ for all eight focus locations. We chose to plot against $L_{z}$ because it was in most cases larger than $L_{x}$ and $L_{y}$. The vertical extent $L_{z}$ ranged from 4 to $28 \mathrm{~mm}$, with larger $L_{z}$ tending to coincide with higher temperatures. The range of $T\left(\mathbf{r}_{f i}\right)$ was $41.8-44.8^{\circ} \mathrm{C}$. The range of $T\left(\mathbf{r}_{p i}\right)$ was $42.3-44.8^{\circ} \mathrm{C}$. Six of the eight focus locations had $T\left(\mathbf{r}_{f i}\right) \geqslant 42.0^{\circ} \mathrm{C}$.

In general, raising the focus temperature results in a larger heating zone. For the case of $\mathbf{r}_{f 1}$, if we re-scale $P$ so that $T\left(\mathbf{r}_{f 1}\right)=42.5^{\circ} \mathrm{C}$, then the new heating zone extents are $L_{z}=13, L_{x}=8$, and $L_{y}=7 \mathrm{~mm}$. In a clinical setting, re-scaling $P$ in this way may be useful in balancing the benefits of a high focus-location temperature with the benefits of a desired heating zone size.

\subsection{6 year old patient, mismatched beamformer with no suppression points}

Next we consider the performance of the beamformer when there is a mismatch between the assumed and actual dielectric properties of the patient. For the same eight focus locations, we 

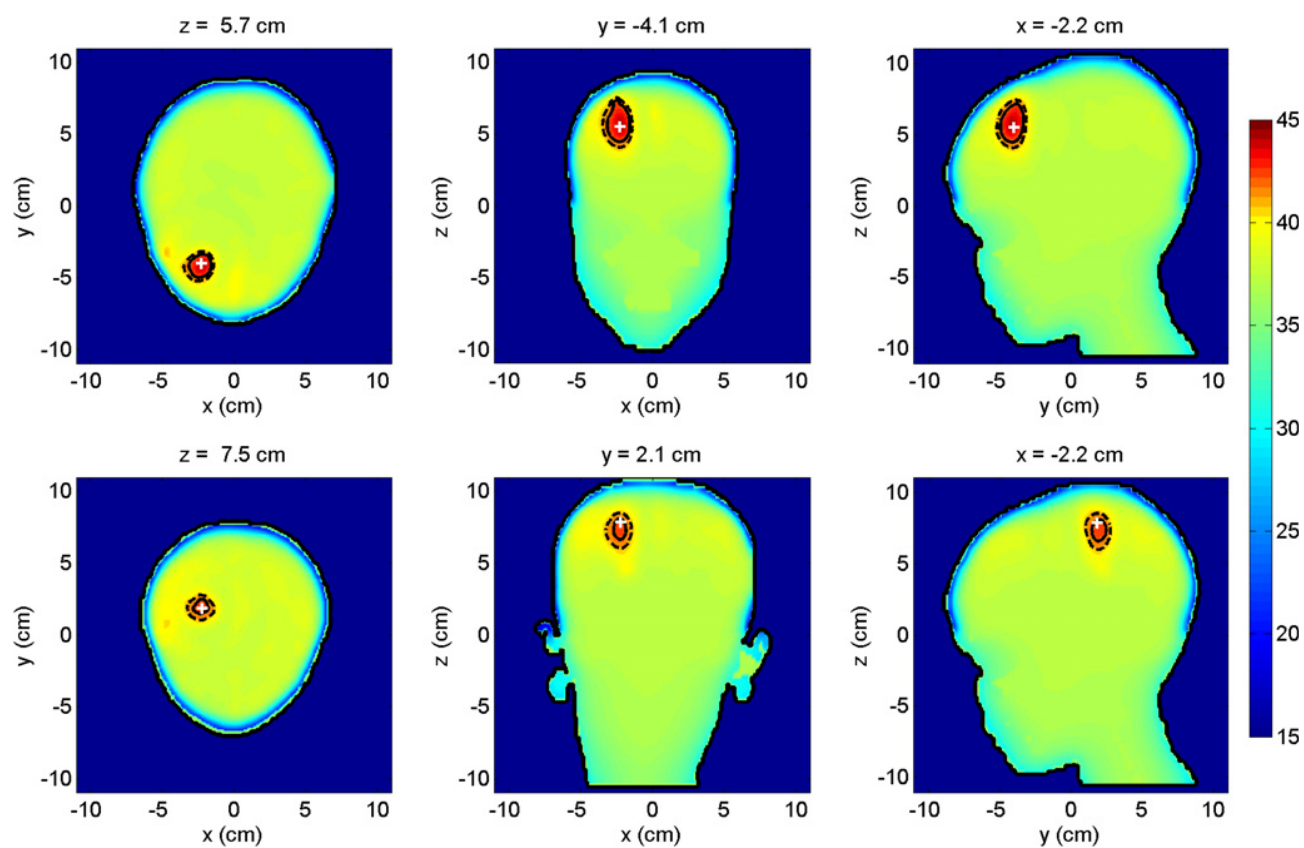

Figure 2. Steady-state temperature distributions through the maximum temperature location in the 6 year old patient for (top) $\mathbf{r}_{f 1}(-2.2,-4.0,5.5) \mathrm{cm}$ and (bottom) $\mathbf{r}_{f 2}(-2.2,1.8,7.9) \mathrm{cm}$. The white cross-hairs mark the projection of the focus onto each orthogonal cross-section. The solid line depicts the heating zone $\left(T>42{ }^{\circ} \mathrm{C}\right)$, while the dashed line is a $41{ }^{\circ} \mathrm{C}$ contour. $T\left(\mathbf{r}_{f 1}\right)=$ $44.0^{\circ} \mathrm{C}$, while $T\left(\mathbf{r}_{f 2}\right)=42.2^{\circ} \mathrm{C}$.

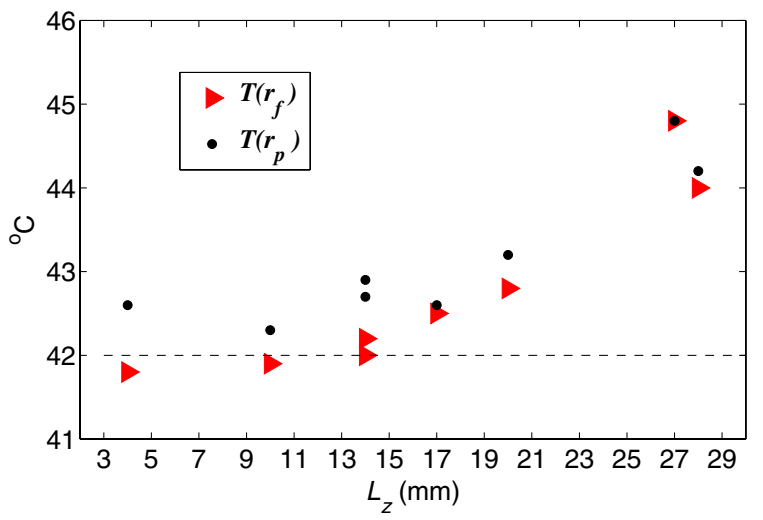

Figure 3. Peak and focus-location temperatures $\left(T\left(\mathbf{r}_{p}\right)\right.$ and $T\left(\mathbf{r}_{f}\right)$, respectively) as a function of the vertical extent of the heating zone for the eight focus locations in the 6 year old patient.

obtained $\mathbf{h}\left(\mathbf{r}_{f i}\right)$ from a treatment planning simulation wherein $\epsilon_{r}$ and $\sigma_{\text {eff }}$ for each tissue type were increased by $10 \%$ relative to the actual properties of the virtual patient. We chose $10 \%$ as a representative level of uncertainty based on $1 \mathrm{GHz}$ data for gray matter (Gabriel 1996), the single largest constituent tissue in the region surrounded by the array. We applied an equal 

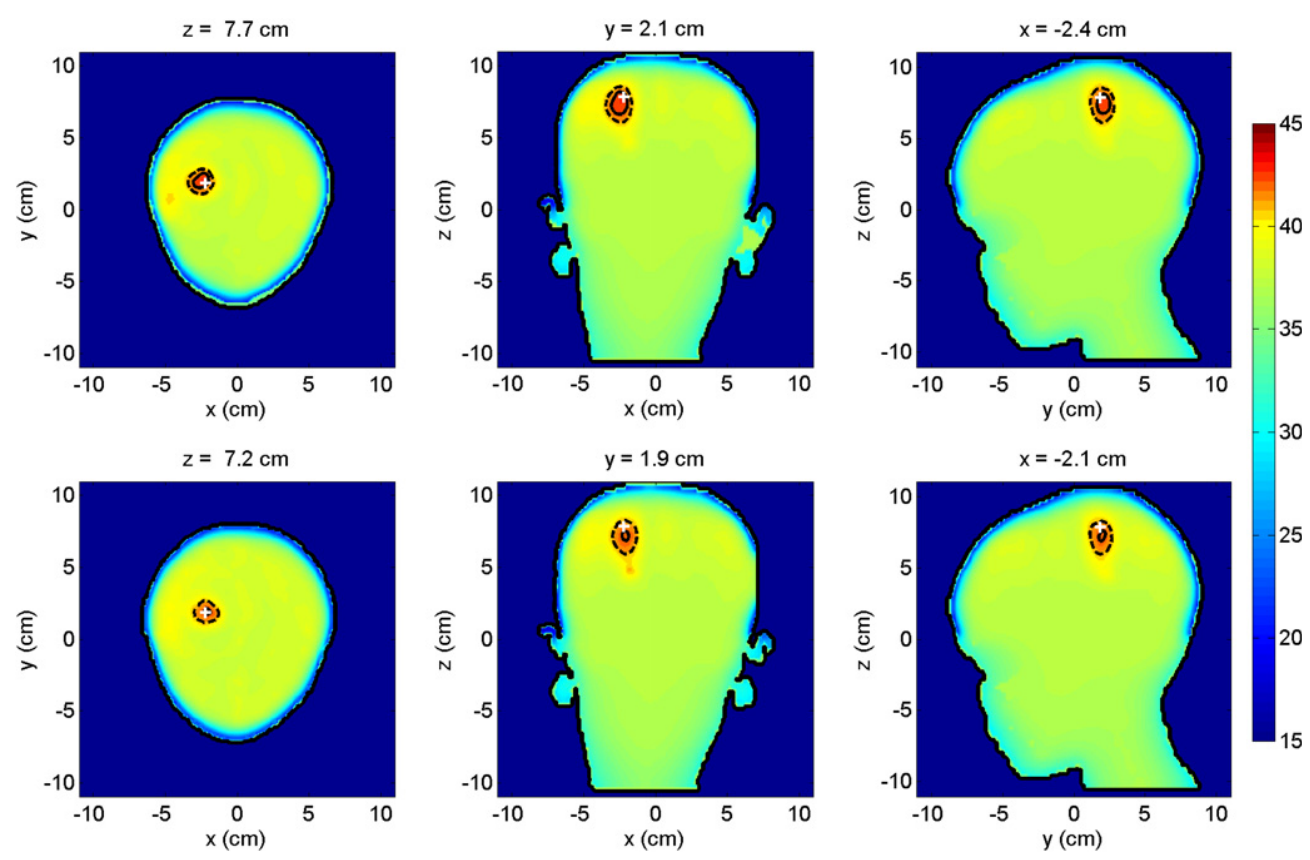

Figure 4. Steady-state temperature distributions for $\mathbf{r}_{f 2}(-2.2,1.8,7.9) \mathrm{cm}$ for the 6 year old patient. For the top row, the assumed dielectric properties of the patient were $10 \%$ higher than the properties used for performance evaluation. $T\left(\mathbf{r}_{f 2}\right)=42.1{ }^{\circ} \mathrm{C}$ (top row). For the bottom row, the assumed dielectric properties of the patient were $10 \%$ lower than the properties used for performance evaluation. $T\left(\mathbf{r}_{f 2}\right)=41.6{ }^{\circ} \mathrm{C}$ (bottom row). The cross-hairs, the solid line, and the dashed line have the same connotation as in figure 2.

mismatch to all voxels in order to obtain relatively severe propagation model error for a given level of mismatch.

The weights $\mathbf{w}$ were then chosen according to (1). To test the effectiveness of this mismatched beamformer, we conducted a performance evaluation simulation using the original phantom (with the unaltered dielectric properties). The steady-state thermal distribution for $\mathbf{r}_{f 2}$ is given in figure 4 (top row). The distribution is very similar to the distribution obtained from the matched case that is shown in figure 2 (bottom row).

We also designed a beamformer using a model wherein $\epsilon_{r}$ and $\sigma_{\text {eff }}$ were decreased by $10 \%$ and evaluated its performance. The thermal distribution for $\mathbf{r}_{f 2}$ is shown in figure 4 (bottom row). The heating zone for $\mathbf{r}_{f 2}$ is considerably smaller. The use of underestimated dielectric properties in the treatment planning simulation has degraded the focus and exacerbated a potential hot spot near the target, requiring $P$ to be scaled down so that the hot spot falls in the temperature range $40.95 \pm 0.15^{\circ} \mathrm{C}$.

The effect of a dielectric properties' mismatch on the size of the heating zone is shown in figure 5 for all eight points. Overestimating the dielectric properties in the treatment planning simulation had a negligible effect on $L_{z}$ for seven of the eight focus locations. In the case of $\mathbf{r}_{f 4}$, we observed a significantly larger $L_{z}$. Conversely, underestimating the dielectric properties in the treatment planning simulation resulted in $L_{z}=0$ for two cases $\left(\mathbf{r}_{f 4}, \mathbf{r}_{f 7}\right)$. This signifies that no heating zone $\left(T>42{ }^{\circ} \mathrm{C}\right)$ could be created without also creating hot spots. These results suggest that the beamforming algorithm is robust to modest overestimation of dielectric 


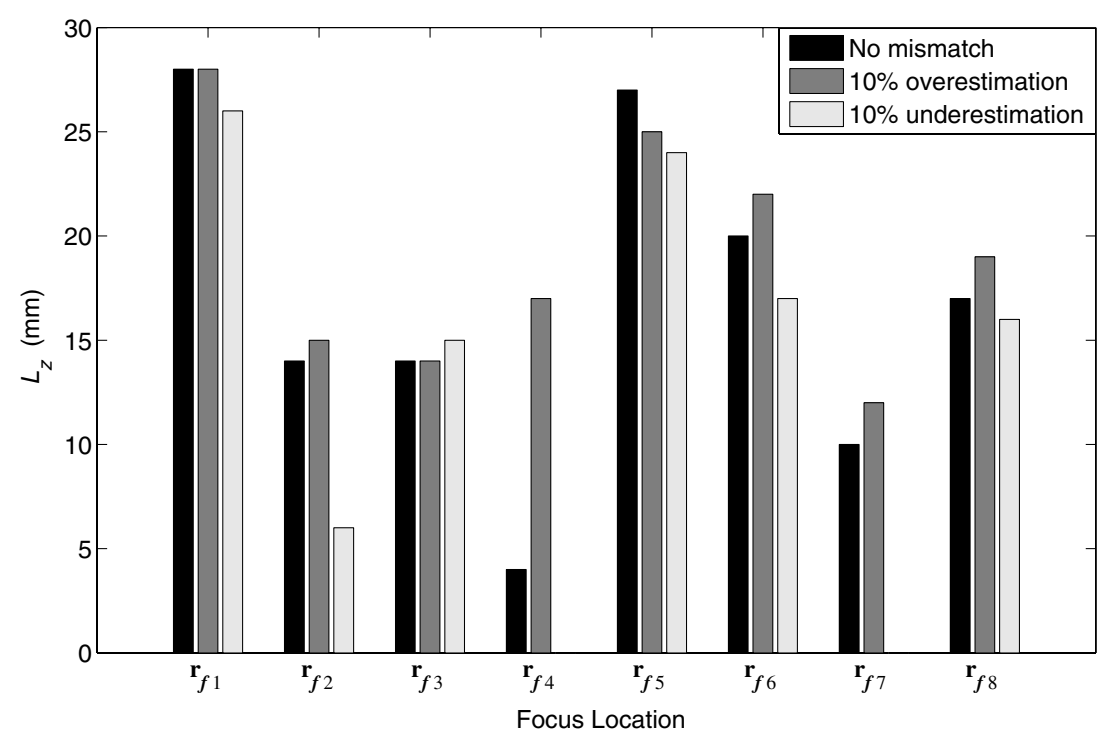

Figure 5. The vertical extent of the heating zone in the model of the 6 year old patient when the dielectric properties assumed in the beamformer design are equal to $10 \%$ over and $10 \%$ under the actual properties. Underestimation of dielectric properties results in $L_{z}=0 \mathrm{~mm}$ (no heating zone) for $\mathbf{r}_{f 4}$ and $\mathbf{r}_{f 7}$.

properties and inconsistent for modest underestimation of dielectric properties. This finding is consistent with Zastrow et al (2010).

\subsection{1 year old patient, matched beamformer with suppression}

Lastly, we consider the suppression of hot spots using an iterative design procedure. We chose to use the numerical model of the 11 year old patient for this study, as the model's higher CSF levels potentially lead to more hot spots. The 11 year old patient has $177 \mathrm{~mL}$ of CSF in the brain. The 6 year old patient has similar head dimensions as the 11 year old patient, but only $151 \mathrm{~mL}$ of CSF in the brain.

One focus location was chosen at random using the same CSF and depth constraints as were used for the previous eight focus locations in the 6 year old's head. This focus location, denoted as $\mathbf{r}_{f}$, has coordinates $(2.1,-2.0,3.9) \mathrm{cm}$. The propagation function $\mathbf{h}\left(\mathbf{r}_{f}\right)$ was obtained using the exact dielectric properties. The weights $\mathbf{w}$ were designed according to (1) assuming no suppression points. Steady-state temperature slices through $\mathbf{r}_{p}$ (the location of peak temperature) are shown in figure 6 (top row). Note that $P$ has again been scaled so that the maximum $T\left(\mathbf{r}_{f}\right)$ is achieved (in this case, $41.2{ }^{\circ} \mathrm{C}$ ) without creating hot spots.

We identified the hottest point exterior to the $41{ }^{\circ} \mathrm{C}$ contour surrounding $\mathbf{r}_{p i}$ as the first suppression point, denoted as $\mathbf{r}_{s 1}$. We obtained the propagation function, $\mathbf{h}\left(\mathbf{r}_{s 1}\right)$, for this suppression point. The weights $\mathbf{w}$ were re-designed according to (1) with $\alpha_{1}=200$. After using one suppression point, the maximum $T\left(\mathbf{r}_{f}\right)$ such that no hot spots occurred decreased by $0.1{ }^{\circ} \mathrm{C}$.

We then repeated the design procedure described above four times. For each iteration, we added a new suppression point while keeping all previous suppression points. The suppression point $\mathbf{r}_{s n}$ was chosen to be the hottest point external to the $41{ }^{\circ} \mathrm{C}$ contour surrounding $\mathbf{r}_{p i}$ 

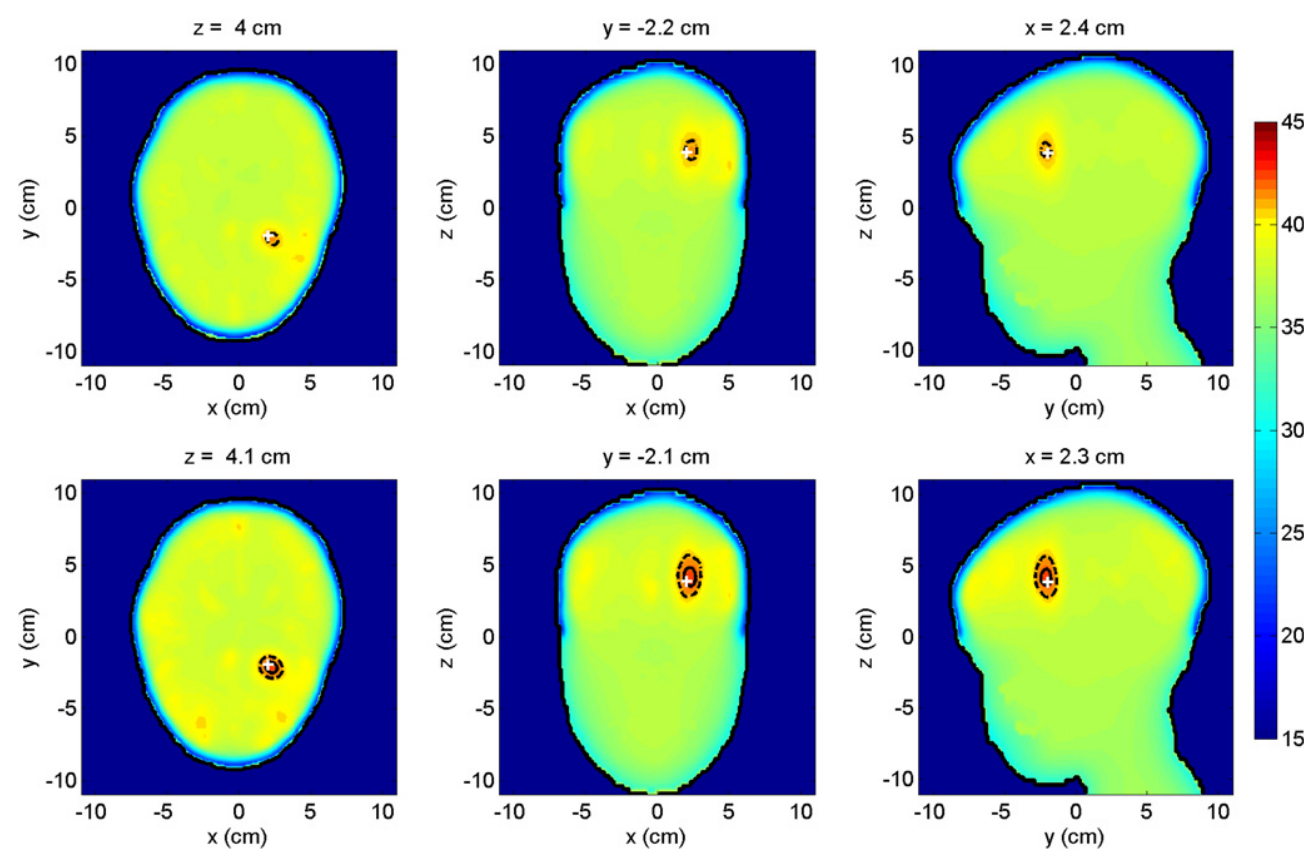

Figure 6. Steady-state temperature distributions for $\mathbf{r}_{f}(2.1,-2.0,3.9) \mathrm{cm}$ in the 11 year old patient. For the top row, no suppression points were used, and $T\left(\mathbf{r}_{f}\right)=41.2^{\circ} \mathrm{C}$. For the bottom row, five suppression points were used, and $T\left(\mathbf{r}_{f}\right)=42.2^{\circ} \mathrm{C}$. The cross-hairs, the solid line, and the dashed line have the same connotation as in figure 2.

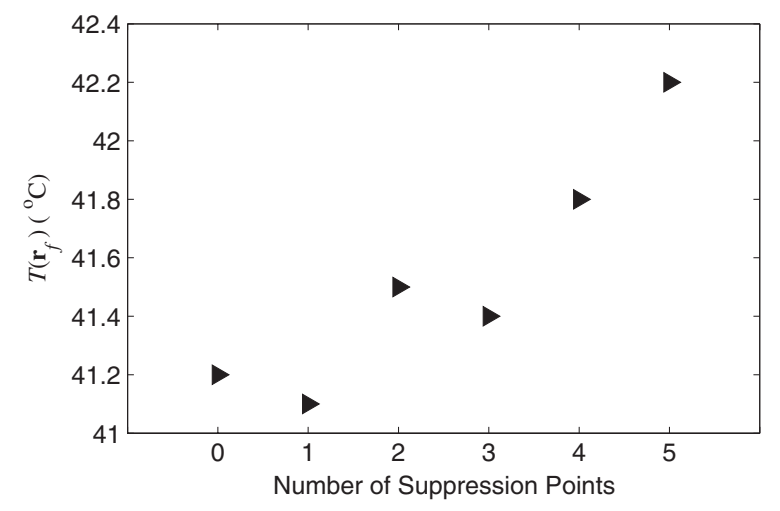

Figure 7. Focus-location temperature as a function of the number of suppression points for $\mathbf{r}_{f}$ $(2.1,-2.0,3.9) \mathrm{cm}$ in the 11 year old patient.

after $n-1$ iterations. Each suppression point weight, $\alpha_{n}$, was chosen to be 200. The focus temperature is plotted by iteration in figure 7. The maximum $T\left(\mathbf{r}_{f}\right)$ such that no hot spots are created reaches $42.2{ }^{\circ} \mathrm{C}$ after the last iteration, which is $1.0^{\circ} \mathrm{C}$ higher than could be achieved with no suppression points. Figure 6 (bottom row) shows steady-state thermal slices after the last iteration. We see that decreasing energy deposition at the five suppression points came at the expense of increasing the temperature at other locations in the brain. 


\subsection{Practical issues}

The results reported above suggest that the patient-specific, focus-location-specific microwave beamforming approach shows promise for non-invasive hyperthermia treatment of pediatric brain cancer. Further work needs to be done in order to assess the feasibility of realizing a practical system. Practical issues include designing compact, efficient antenna elements and determining the optimal array configuration and coupling medium. Electrically small, omnidirectional voltage sources are useful in evaluating the potential performance of the proposed beamforming strategy. However, these sources would be too inefficient for a clinical hyperthermia system. Techniques for shielding the patient's eyes and the environment outside the array from the microwave radiation will also need to be developed.

The results of this study establish the feasibility of creating heating zones at desired focus locations in the brain while maintaining temperatures below $41{ }^{\circ} \mathrm{C}$ elsewhere. Depending on the focus location and the propagation environment for a particular patient, regions of relatively high temperature (up to $41^{\circ} \mathrm{C}$ ) may still be created at locations in the brain where such a temperature may be unsafe. The physician must weigh the benefits and risks of treatment in considering whether microwave hyperthermia treatment is appropriate for each patient. Our preliminary investigation suggests that beamforming may offer the flexibility in treatment planning to accommodate input from the physician regarding regions to safeguard through suppression.

\section{Conclusion}

This numerical study establishes the feasibility of an array-based microwave transmitbeamforming approach for hyperthermia treatment of pediatric brain tumors. The beamforming approach is shown to be feasible despite the prevalence of lossy high-watercontent tissue and CSF in the brain. The beamformers are designed according to patientspecific and focus-location-specific propagation data, and operate at $1 \mathrm{GHz}$ in order to balance resolution with penetration depth. Beamformer performance is evaluated for a set of randomly chosen focus points in an MRI-derived human head model using electromagnetic FDTD simulations to determine power deposition and thermal FDTD simulations to determine the steady-state temperature distribution. The results show that localized heating zones can be created in the child head model for focus locations that are not located near significant amounts of CSF. We show that the beamforming approach is robust to modest overestimation of dielectric properties in the propagation model. The beamforming approach is less robust to modest underestimation of dielectric properties. The ability of the beamformer to suppress unwanted hot spots in a human head model with higher levels of CSF is also demonstrated. Our results suggest that this beamforming technique shows promise for treating pediatric brain tumors non-invasively via focused microwave hyperthermia and that further investigation is warranted.

\section{Acknowledgments}

This work was supported by SEMCAD for Science, the Department of Defense SMART Scholarship for Service Program, the National Science Foundation under grant CMMI0625054, and the Philip D Reed chaired professorship. 


\section{References}

Bernardi P, Cavagnaro M, Pisa S and Piuzzi E 2003 Specific absorption rate and temperature elevation in a subject exposed in the far-field of radio-frequency sources operating in the 10-900-MHz range IEEE Trans. Biomed. Eng. 50 295-304

Converse M, Bond E, Van Veen B and Hagness C 2006 A computational study of ultra-wideband versus narrowband microwave hyperthermia for breast cancer treatment IEEE Trans. Microw. Theory Tech. 54 2169-80

Duck F A 1990 Physical Properties of Tissue (New York: Academic)

Duffner P 2004 Long-term effects of radiation therapy on cognitive and endocrine function in children with leukemia and brain tumors Neurologist 10 293-310

Duffner P, Cohen M, Thomas P and Lansky S 1985 The long-term effects of cranial irradiation on the central nervous system Cancer 56 1841-6

Dunn D, Rappaport M and Terzuoli A Jr 1996 FDTD verification of deep-set brain tumor hyperthermia using a spherical microwave source distribution IEEE Trans. Microw. Theory Tech. 44 1769-77

Gabriel C 1996 Compilation of the dielectric properties of body tissues at RF and microwave frequencies Technical Report Occupational and Environmental Health Directorate, Radiofrequency Radiation Division, Brooks AFB, TX

Gordon R, Roemer R and Horvath S 1976 A mathematical model of the human temperature regulatory system? Transient cold exposure response IEEE Trans. Biomed. Eng. vol BME-23 pp 434-44

Gouzouasis I, Karanasiou I and Uzunoglu N 2007 FDTD study of the focusing properties of a hybrid hyperthermia and radiometry imaging system using a realistic human head model 29 th Ann. Int. Conf. of the IEEE Engineering in Medicine and Biology Society, 2007 (EMBS 2007) pp 3552-5

Harima Y, Nagata K, Harima K, Ostapenko V, Tanaka Y and Sawada S 2001 A randomized clinical trial of radiation therapy versus thermoradiotherapy in stage IIIB cervical carcinoma Int. J. Hyperth. 17 97-105

Kapp D 1996 Efficacy of adjuvant hyperthermia in the treatment of superficial recurrent breast cancer: confirmation and future directions Int. J. Radiat. Oncol. Biol. Phys. 35 1117-21

Karanasiou I, Karathanasis K, Garetsos A and Uzunoglu N 2008 Development and laboratory testing of a noninvasive intracranial focused hyperthermia system IEEE Trans. Microw. Theory Tech. 56 2160-71

Overgaard J, Bentzen S, González D, Hulshof M, Arcangeli G, Dahl O and Mella O 1995 Randomised trial of hyperthermia as adjuvant to radiotherapy for recurrent or metastatic malignant melanoma Lancet 345 540-3

Packer R, Sutton L, Atkins T, Radcliffe J, Bunin G, D’Angio G, Siegel K and Schut L 1989 A prospective study of cognitive function in children receiving whole-brain radiotherapy and chemotherapy: 2-year results J. Neurosurg. 70 707-13

Pollack I 1999 Pediatric brain tumors Semin. Surg. Oncol. 16 73-90

Sneed P, Stauffer P, Li G and Stege G 2004 Hyperthermia Textbook of Radiation Oncology 2nd edn (Pennsylvania, PA: Saunders) pp 1569-96

Sneed $\mathrm{P}$ et al 1998 Survival benefit of hyperthermia in a prospective randomized trial of brachytherapy boost $+/-$ hyperthermia for glioblastoma multiforme Int. J. Radiat. Oncol. Biol. Phys. 40 287-95

Taflove A and Hagness S C 2005 Computational Electrodynamics: The Finite-Difference Time-Domain Method 3rd edn (Norwood, MA: Artech House Publishers)

Tanaka R, Kim C, Yamada N and Saito Y 1987 Radiofrequency hyperthermia for malignant brain tumors: preliminary results of clinical trials Neurosurgery 21 478-83

Van der Zee J, González D, Van Rhoon G, van Dijk J, Van Putten W and Hart A 2000 Comparison of radiotherapy alone with radiotherapy plus hyperthermia in locally advanced pelvic tumours: a prospective, randomised, multicentre trial Lancet 355 1119-25

Winter A, Laing J, Paglione R and Sterzer F 1985 Microwave hyperthermia for brain tumors Neurosurgery 17 387-99

Zastrow E, Hagness S and Van Veen B 2010 3D computational study of non-invasive patient-specific microwave hyperthermia treatment of breast cancer Phys. Med. Biol. 55 3611-29 